\title{
Hypoxia Inducible Factor 1-a in Acute Ischemic Stroke: Good Predictor for Clinical Outcome
}

\author{
Yeremia Tatang \\ Siloam Hospitals Kebon Jeruk \\ Henny Anggraini Sadeli \\ Universitas Padjadjaran \\ Ida Parwati \\ Universitas Padjadjaran \\ Ahmad Rizal \\ Universitas Padjadjaran \\ Ramdan Panigoro \\ Universitas Padjadjaran
}

Lisda Amalia ( $\nabla$ dr.lisda@gmail.com )

Universitas Padjadjaran https://orcid.org/0000-0001-5676-0787

\section{Research article}

Keywords: acute ischemic stroke, HIF-1a, NIHSS

Posted Date: April 9th, 2019

DOI: https://doi.org/10.21203/rs.2.1865/v1

License: (c) (i) This work is licensed under a Creative Commons Attribution 4.0 International License.

Read Full License 


\section{Abstract}

Background. Stroke is the third leading causes of death and can cause severe disability. Ischemic stroke has a higher prevalence compared to hemorrhage stroke. Hypoxia-inducible factor- $1 \mathrm{a}(\mathrm{HIF-1a})$ is a transcription factor which maintains cellular homeostasis in response to hypoxia. It can trigger apoptosis while stimulating angiogenesis process and decrease neurological deficit after an ischemic stroke. However, this protein complex has not been widely investigated. Objective. Here, we examined the potential of HIF-1a as a marker for neuroplasticity process after ischemic stroke. Methods. Serum HIF-1a were measured in acute ischemic stroke patients. National Institute of Health Stroke Scale (NIHSS) were assessed on the admission and discharge day (between days 7 and 14). To classify the ischemic stroke, we used (Trial of Org 10172 in Acute Stroke Treatment) TOAST criteria. Statistical significances were calculated with Spearman rank test. Results. A total of 58 patients, 31 with large artery atherosclerosis LVD and 27 with small vessel disease (SVD) were included in this study. HIF-1 a level in LVD group was (mean \pm SD) $0.5225 \pm 0.2459 \mathrm{mg} / \mathrm{L}$ and in SVD group was $0.3815 \pm 0.121 \mathrm{mg} / \mathrm{L}$. HIF-1 a was higher $(\mathrm{p}=$ 0.004 ) in LVD group than in SVD group. The initial NIHSS score in LVD group was (mean \pm SD) $15.46 \pm$ 2.61 and discharge NIHSS score was $13.31 \pm 3.449$. Initial NIHSS score in SVD group was $6.07 \pm 1.82$ and the discharge NIHSS was $5.703 \pm 1.7055$. In LVD group, HIF-1 a was correlated significantly with initial NIHSS $(p=0.0000)$ and discharge NIHSS $(p=0.0000, r=0.93)$. This was also the case for SVD. We found a significant correlation between the level of HIF-1 a with initial NIHSS $(p=0.0000)$ and discharge NIHSS ( $p=0.0383)$ in SVD group $(r=0.94)$. Conclusion. HIF-1a has a strong correlation with NIHSS and it may be used as the predictor of acute ischemic stroke outcome.

\section{Introduction}

Stroke is the third leading causes of mortality and severe disability worldwide. Ischemic stroke is responsible for around eighty percent of strokes. It occurs as the arteries narrow or become blocked, leading to severe reduction in the blood flow or ischemia. It is a medical emergency and timely management is crucial to minimize neuronal damage and potential complications.1,2 Following a hypoxic condition, brain responses it by initiating gene expressions that function in preconditioning hypoxia. One of the transcription factors involved is the Hypoxia-inducible factor-1a (HIF-1a).3,4,5

HIF-1a is a transcription activator that plays an integral role to maintain homeostasis after a hypoxic insult. A disproportion in the supply and oxygen demand in cerebral tissue promotes a sequence of biochemical and molecular events that leads in neuron cell death. In response to such a detrimental situation, the tissue adopt several cellular mechanisms. One of recently studied mechanism is the induction of HIF-1a.4,6,7,11 It is a transcriptional regulator of oxygen stability and a main stimulator of adaptive response by upregulating several target genes which are important in erythropoiesis, angiogenesis, glucose transport and metabolism.7,8,9,13 However, studies also suggested the role HIF-1a in the apoptotic cascade by stimulating pro-apoptotic molecules namely Nix, IL 20 and Bnip30 which results in mitochondrial dysfunction and leads to neuronal cell death.7,12,13 Therefore, deeper understanding regarding the role of HIF-1a, specifically in ischemic stroke, is required to improve recovery 
and brain repair process after stroke. To date, studies regarding the relationship of HIF-1a and ischemic stroke were all conducted in animal model. To our knowledge, our study was the first study to determine the association between HIF-1a, the severity of stroke ischemia, and its outcome in human.

\section{Methods}

This was a cross-sectional study on acute ischemic stroke patients which was confirmed by non-contrast brain computerized tomography (CT) less than 24 hours onset and we collected HIF-1a level from blood serum on admission. We assessed the neurological deficit using the National Institute of Health Stroke Scale (NIHSS) in the admission and discharge day (between days 7 and 14). Ischemic stroke classification were established using Trial of Org 10172 in Acute Stroke Treatment (TOAST) criteria. We classified acute ischemic stroke patients into two groups according to TOAST stroke classification: large vessel disease (large atherosclerosis and cardioembolic stroke) or LVD and small vessel disease (lacunar stroke) or SVD. We excluded patients with chronic renal failure, acute myocardial infarction, severe respiratory failure, severe anemia, dementia, acute limb ischemia and history of brain injury. To determine statistical significances, Spearman rank test were performed. $P$ value $(p)$ below 0.001 is considered significant. R coefficient was calculated to establish the correlation.

\section{Results}

A total of 58 patients (31 with LVD and 27 with SVD) were eligible in this study. The mean age of our subjects were (mean \pm SD) $59.52 \pm 11.52$ years. We collected laboratory data such as blood glucose, uric acid, renal and lipid profile (table 1 ).

HIF-1a level in LVD group was (mean \pm SD) $0.5225 \pm 0.2459 \mathrm{mg} / \mathrm{L}$ and SVD group was $0.3815 \pm 0.121$ $\mathrm{mg} / \mathrm{L}$. The level of HIF-1 a was significantly higher $(p=0.004)$ in the LVD group than in the SVD group (table 2 and figure 1).

LVD or SVD type were found to correspond with the size of ischemic lesions identified from our noncontrast brain CT results (supplementary data). Wide ischemic changes were found in the LVD group. The initial NIHSS score in LVD group was (mean \pm SD) $15.46 \pm 2.61$ and discharge NIHSS score was $13.31 \pm 3.449$. Initial NIHSS score in SVD group was $6.07 \pm 1.82$ and the discharge NIHSS was $5.703 \pm$ 1.7055 (table 3).

In the LVD group, the level of HIF-1a was significantly correlated with initial NIHSS $(p=0.0000)$ and discharge NIHSS $(p=0.0000)$. In the SVD group we identified a similar result, the level of HIF-1a significantly correlated with initial NIHSS $(p=0.0000)$ and discharge NIHSS $(p=0.0383)$.

Table 4 showed the correlation between HIF-1a level and NIHSS in both groups. Here, we identified a strong correlation between the two variables in both groups.

\section{Discussion}


Our study include 58 acute ischemic stroke subjects who were divided into LVD and SVD groups according to TOAST criteria. For the first time in human study, we identified an association between HIF1a level and the subtypes of ischemic stroke. The level of HIF-1a (table 2 and figure 1) was significantly higher in the LVD group than in the SVD group $(p=0.004)$. This result was similar with previous studies conducted in animal model of cerebral ischemia.3

In addition, from our non-contrast brain CT results, we identified a relation between the groups with the size of ischemic lesions (supplementary data). Wide ischemic changes were found in the LVD group. This finding showed the extent of neuronal brain damage after ischemic stroke.

This result was in accordance to previous studies which suggest that a wide brain damage is associated with an increase of HIF-1a production. The production of HIF-1a is initiated in the preconditioning-hypoxia sequence induced by neuronal damage.7,8,9,11,12,13

A wide brain damage also correlates with worsening of neurological deficit in acute ischemic stroke patients. 4 In this study we found that the initial NIHSS score in the LVD group was $15.46 \pm 2.61$ and discharge NIHSS score was $13.31 \pm 3.449$. Initial NIHSS score in SVD group was $6.07 \pm 1.82$ and the discharge NIHSS was $5.703 \pm 1.7055$ (table 3 ).

In this study, we established an association between HIF-1 a and NIHSS (table 4). There were strong correlations between HIF-1a and on admission and discharge NIHSS in both groups. In previous studies in mice and rats, HIF-1a was activated in a biphasic manner lasting up to ten days after a hypoxic stimulation3, stimulates pro-angiogenic factors4, promototes neuronal viability 7 , and ameliorates brain injury13. We examined the HIF-1a level only on admission but as it correlated significantly with the NIHSS, we may suggest that its level would reduce on discharge day and is associated with the improvement of clinical outcome, as occurs in mice model. Future study examining serial HIF-1a level needs to be performed to prove this hypothesis.

From this study we conclude that HIF-1 a may be used to predict the clinical outcome in acute ischemic stroke patients. Further study to established its precise predictive value needs to be conducted.

In addition, drug and molecule which activate HIF-1a in vitro and in vivo was proved to own a neuroprotective effect in mice cerebral ischemia model.3,8,9 Therefore the long run, development of molecule regulator for HIF-1 a stability and signalling in human is needed to produce a therapeutic option which enhance recovery and repair after ischemic stroke.

\section{Conclusion}

HIF-1a level was associated with severity of ischemic lesion, large vessel disease had a higher level of HIF-1a compared with small vessel disease. HIF-1a had a strong correlation with NIHSS, both on admission and patients' discharge. From our findings, we can conclude that HIF-1a may be a good predictor for a better clinical outcome in acute ischemic stroke patients. 


\section{List Of Abbreviation}

HIF 1-a: Hypoxia Inducible Factor-1 alfa; NIHSS: National Institute of Health Stroke Scale; SVD: Small Vessel Disease; LVD: Large Vessel Disease; CT: Computed Tomography; TOAST: Trial of Org 10172 in Acute Stroke Treatment; IL-20: Interleukin 20; Bnip 30: Bcl-2/E1B-19K-interacting protein 30

\section{Declaration}

Ethical approval of this study were obtained from Research Ethic Committee of Hasan Sadikin Hospital Bandung. All data or analysis during this study are provided by the corresponding author in this article supplementary files.

\section{Competing Interest}

There is no competing interest.

\section{Funding}

This research was funded by Hibah Internal Unpad 2016, which is an internal grant for lecturer in Padjadjaran University to fund their research. This funding supported data collection, analysis, and interpretation of the data.

\section{Ethical Clearance}

This research was granted approveal by the ethical committee Dr. Hasan Sadikin Hospital Bandung, Indonesia (LB.04.01/A05/EC/069/II/2016).

\section{Consent to Participate}

Consent to participate was obtained from all participants. A consent form was written and signed by each participant. The ethics committee has approved this procedure.

\section{Consent for Publication}

This publication is one of the requirements to pursue doctoral title from Faculty of Medicine, University of Padjadjaran Bandung, Indonesia.

\section{Availability of Data and Material}

The full data are showed in supplementary material.

\section{Authors' Contribution}

Lisda Amalia : Preparation, collecting, analysis, making of manuscript, submission for publication 
Yeremia Tatang: Preparation, collecting, analysis

Henny A. Sadeli : Analysis

Ida Parwati : Analysis

Ahmad Rizal : Analysis

Ramdan Panigoro : Analysis

\section{References}

1. Petito CK (ed). The Neuropathology of Focal Brain Ischemia. Basel: ISN Neuropath Press; 2005: 21521.

2. Takasawa M, Beech JS, Fryer TD, Hong YT, Hughes JL, Igase $\mathrm{K}$, et al. Imaging of brain hypoxia in permanent and temporary middle cerebral artery occlusion in the rat using F-18-fluoromisonidazole and positron emission tomography: a pilot study. J Cereb Blood FlowMetab. 2007; 27:679-89

3. Baranova O, Miranda LF, Pichiule P, Dragatsis I, Johnson RS, Chavez JC. Neuron-specific inactivation of the hypoxia inducible factor 1 alpha increases brain injury in a mouse model of transient focal cerebral ischemia. J Neurosci. 2007; 27:6320-32

4. Li Y, Dong H, Chen M, Liu J, Yang L, Chen S, et al. Preconditioning with repeated hyperbaric oxygen induces myocardial and cerebral protection in patients undergoing coronary artery bypass graft surgery: A prospective, randomized, controlled clinical trial. J Cardiothorac Vasc Anesth. 2011;25:908-16.

5. Biswas S, Charlesworth PJ, Turner GD, et al. CD31 angiogenesis and combined expression of HIF1alpha and HIF-2alpha are prognostic in primary clear-cell renal cell carcinoma (CC-RCC), but HIF alpha transcriptional products are not: implications for antiangiogenic trials and HIF alpha biomarker studies in primary CC-RCC. Carcinogenesis. 2012;33(9):1717-1725.

6. Kulik T, Kusano Y, Aronhime S, Sandler AL, Winn HR. Regulation of cerebral vasculature in normal and ischemic brain. Neuropharmacology. 2008 55: 281-288.

7. Vangeison G, Carr D, Federoff HJ, Rempe DA. The good, the bad, and the cell type-specific roles of hypoxia inducible factor-1 alpha in neurons and astrocytes. J Neurosci. 2008;28:1988-1993.

8. Bruick RK. Oxygen sensing in the hypoxic response pathway: regulation of the hypoxia-inducible transcription factor. Genes Dev. 2003;17:2614-2623

9. Giusti S, Plazas SFD. Neuroprotection by hypoxic preconditioning involves upregulation of hypoxiainducible factor-1 in a prenatal model of acute hypoxia. J Neurosci Res. 2011;22766:468- 478 
10. McLaren AT, Marsden PA, Mazer CD, Baker AJ, Stewart DJ, Tsui AK, Li X, Yucel Y, Robb M, Boyd SR, Liu E, Yu J, Hare GM. Increased expression of HIF-1 alpha, nNOS, and VEGF in the cerebral cortex of anemic rats. Am J Physiol Regul Integr Comp Physiol. 2007;292:R403-R414

11. Mehta SL, Manhas N, Raghubir R. Molecular targets in cerebral ischemia for developing novel therapeutics. Brain Res Rev. 2007;54:34-66

12. Stolze IP, Tian YM, Appelhoff RJ, Turley H, Wykoff CC, Gleadle JM, Ratcliffe PJ. Genetic analysis of the role of the asparaginyl hydroxylase factor inhibiting hypoxia-inducible factor $(\mathrm{FIH})$ in regulating hypoxia-inducible factor (HIF) transcriptional target genes. J Biol Chem. 2004;279:42719-42725

13. Shi Q, Zhang P, Zhang J, Chen X, Lu H, Tian Y, Parker T L, Liu Y. Adenovirus-mediated brain-derived neurotrophic factor expression regulated by hypoxia response element protects brain from injury of transient middle cerebral artery occlusion in mice. Neurosci Lett. 2009;465:220- 225

\section{Tables}

Table 1. Demographic and laboratory data

\begin{tabular}{|c|c|c|c|c|c|}
\hline No & Variable & Mean & SD & Min & Max \\
\hline \multicolumn{6}{|c|}{ Demographic } \\
\hline 1 & Age & 59.53 & 11.52 & 31 & 91 \\
\hline \multicolumn{6}{|c|}{ Risk factor } \\
\hline 2 & Cholesterol & 200.65 & 45.07 & 83 & 299 \\
\hline 3 & Triglyceride & 123.15 & 60.13 & 49 & 366 \\
\hline 4 & HDL & 46.63 & 16.99 & 17 & 99 \\
\hline 5 & LDL & 141.09 & 38.36 & 60 & 224 \\
\hline 6 & Random Blood Glucose & 141.17 & 60.02 & 77 & 363 \\
\hline 7 & Ureum & 37.52 & 31.16 & 13 & 267 \\
\hline 8 & Kreatinin & 0.93 & 0.32 & 0.38 & 2.4 \\
\hline 9 & Uric acid & 6.23 & 2.13 & 2.1 & 12.4 \\
\hline 10 & $\mathrm{HbA} 1 \mathrm{C}$ & 7.61 & 2.58 & 4.5 & 13.3 \\
\hline
\end{tabular}

Table 2. HIF-1a level in LVD and SVD groups 


\begin{tabular}{llllll} 
HIF-1a & Mean & SD & Min & Max & p \\
\hline Large vessel disease & 0.5225 & 0.2459 & 0.1 & 1.4 & 0.004 \\
\hline Small vessel disease & 0.3815 & 0.121 & 0.1 & 0.6 & \\
\hline
\end{tabular}

\begin{tabular}{lllll} 
NIHSS & Mean & SD & Min & Max \\
\hline Large vessel disease & 16.06 & 2.67 & 11 & 21 \\
On admission & 15.13 & 3.15 & 6 & 20 \\
On discharge & & & & \\
\hline Small vessel disease & 6.07 & 1.82 & 4 & 9 \\
On admission & 5.73 & 1.71 & 4 & 9 \\
On discharge & & & &
\end{tabular}

Table 3. NIHSS During Admission and Discharge Time Points

\begin{tabular}{lcccc} 
NIHSS & Mean & SD & Min & Max \\
\hline Large vessel disease & 16.06 & 2.67 & 11 & 21 \\
On admission & 15.13 & 3.15 & 6 & 20 \\
On discharge & & & & \\
\hline Small vessel disease & & & & \\
On admission & 6.07 & 1.82 & 4 & 9 \\
On discharge & 5.73 & 1.71 & 4 & 9
\end{tabular}

Table 4. Correlation of HIF-1a level and NIHSS

HIF-1a

$$
\text { NIHSS on admission NIHSS on discharge P }
$$

$\mathbf{r}$

\begin{tabular}{lllll}
\hline & $(m e a n \pm S D)$ & $($ mean \pm SD $)$ & & \\
\hline Large Vessel Disease & $16.06 \pm 2.67$ & $15.13 \pm 3.16$ & 0.000 & 0.9299 \\
\hline Small Vessel Disease & $6.07 \pm 1.82$ & $5.7 \pm 1.7$ & 0.00000 & 0.9394
\end{tabular}

\section{Figures}




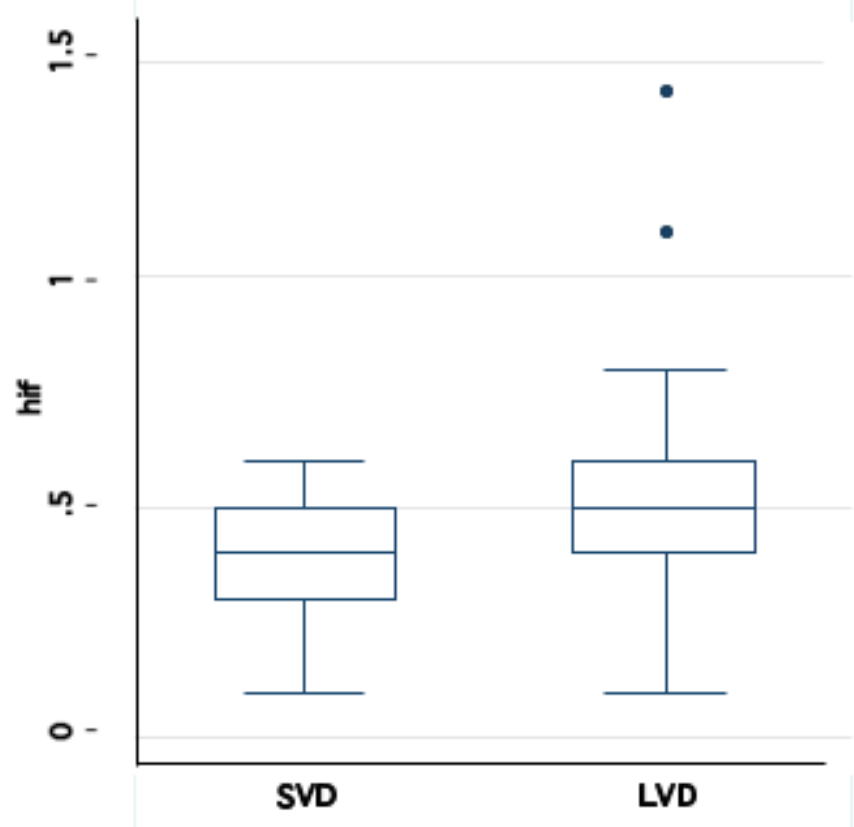

Figure 1

HIF-1a level in SVD and LVD groups 